\title{
Patient and physician discordance of global disease assessment in juvenile dermatomyositis: findings from the Childhood Arthritis \& Rheumatology Research Alliance Legacy Registry
}

Heather Tory ${ }^{1,2^{*}}$ (D), David Zurakowski ${ }^{3}$, Susan $\mathrm{Kim}^{4}$ and the CARRA Juvenile Dermatomyositis Quality Measures Workgroup for the CARRA Registry Investigators

\begin{abstract}
Background: Global disease activity scores (gVAS) capture patient or family (PF) and physician (MD) assessments of disease. This study sought to measure discordance between PF and MD global activity scores in juvenile dermatomyositis (JDM), and determine factors associated with discordance.

Methods: Patients with JDM were included from the Childhood Arthritis and Rheumatology Research Alliance (CARRA) Legacy Registry ( $N=563)$. PF and MD gVAS were assessed for discordance, defined as a $\geq 2$-point difference. Factors associated with discordant gVAS were compared in univariate analysis. Multivariable regression analysis was used to identify predictors of discordance.
\end{abstract}

Results: Almost 40\% ( $N=219)$ of PF and MD gVAS were discordant. Among discordant scores, $68 \%$ of PF rated gVAS $\geq 2$-points above MD, which was associated with calcinosis and lower quality of life and functional scores $(p<0.01)$. MD gVAS rated $\geq 2$-points above PF in 32\%, which was associated with abnormal laboratory results, weakness, arthritis, rash and other skin changes, and current intravenous steroid treatment $(p<0.01)$. In multivariate analysis, predictors for higher PF rating included calcinosis, lower quality of life and functional scores, while predictors for higher MD rating included rash, calcinosis, nailfold capillaroscopy changes, and current intravenous steroid treatment.

Conclusions: Discordance between PF and MD gVAS was common in this JDM cohort. Overall, higher PF rating was associated with poorer patient reported outcome (PRO) scores, while higher MD rating was associated with poorer objective measures. This suggests PF and MD assessments of gVAS may be measuring different aspects of disease, highlighting the importance of integrating PROs into clinical practice and research.

Keywords: Patient reported outcome measures, Juvenile Dermatomyositis, Quality of life, Physician perspective, Patient perspective

\footnotetext{
* Correspondence: htory@connecticutchildrens.org

${ }^{1}$ Division of Pediatric Rheumatology, Connecticut Children's Medical Center,

282 Washington Street, Hartford, CT 06106, USA

${ }^{2}$ Department of Pediatrics, University of Connecticut School of Medicine,

Farmington, CT, USA

Full list of author information is available at the end of the article
}

(c) The Author(s). 2020 Open Access This article is distributed under the terms of the Creative Commons Attribution 4.0 International License (http://creativecommons.org/licenses/by/4.0/), which permits unrestricted use, distribution, and reproduction in any medium, provided you give appropriate credit to the original author(s) and the source, provide a link to the Creative Commons license, and indicate if changes were made. The Creative Commons Public Domain Dedication waiver (http://creativecommons.org/publicdomain/zero/1.0/) applies to the data made available in this article, unless otherwise stated. 


\section{Background}

Juvenile dermatomyositis (JDM) is the most common chronic inflammatory myopathy of childhood [1]. It is a systemic vasculopathy characterized by pathognonomic rashes and proximal muscle weakness. Accurate assessment of disease activity is essential in directing medical care; however, since no single biomarker of disease activity exists for JDM, healthcare providers use a combination of clinical, laboratory and diagnostic measures for assessment. Patient reported outcome measures (PROs) in JDM are not typically monitored in standard clinical practice, despite evidence of the importance of integrating the patient perspective in assessment of disease status [2].

Standardized disease activity measures have been developed by the International Myositis Assessment and Clinical Studies Group (IMACS) and the Pediatric International Trials Organisation (PRINTO), and are recommended for use in all myositis therapeutic trials and clinical studies [3]. Both the IMACS and PRINTO Disease Activity Core Sets Measures include some PROs, including the Patient/Parent Global Activity Assessment Score (PF gVAS), as well as the Physician Global Activity Assessment Score (MD gVAS).

The PF gVAS and MD gVAS are partially validated tools, meant to measure the global evaluation of overall disease activity using a $10 \mathrm{~cm}$ visual analog scale (VAS), where "0"represents no disease activity and "10" represents severe disease activity [4]. These measures are also included in the recently accepted Myositis Response Criteria for JDM, which were developed to define minimal, moderate and major clinical response to treatments in both adult and pediatric myositis, and are recommended for use as primary endpoints in myositis therapeutic trials [5].

Discordance between patient and physician global assessments of disease activity has been reported in several rheumatologic conditions, including rheumatoid arthritis and psoriatic arthritis [6, 7]. Discordance has also been reported between patients/families and physicians in physical function measures in juvenile idiopathic arthritis, with poorer patient/family scores seen in association with poorer scores on PROs, and poorer physician scores associated with poorer objective markers [8]. However, to our knowledge, discordance between patient/family and physician global assessments has not been previously reported in JDM.

In this study, we compared patient/family and physician global assessments of disease activity in JDM and sought to identify predictors of discordance.

\section{Methods}

\section{Setting and study population}

The study population included a cross-sectional cohort of patients with physician-diagnosed JDM enrolled in the North American multi-center Childhood Arthritis and Rheumatology Research Alliance (CARRA) Legacy Registry (CLR) over a 5-year period (2010-2015). A subset of patients with JDM enrolled in this registry has been previously described [9].

Data was abstracted from the baseline enrollment visit using a standardized form. Data included demographics, medication history, PRO measures (global disease activity assessment on a 10 point visual analog scale (VAS), Childhood Health Assessment Questionnaire (CHAQ), overall pain score using a 10 point VAS or the Faces Pain Scale based on age, and overall quality of life scores using a 5 point Likert scale of very poor to excellent) as reported by the patient or the family member (it was not possible to confirm attribution), and physician reported outcome measures (global activity assessment on a 10 point VAS, proximal muscle weakness using a 4 point Likert scale of none, mild, moderate or severe, muscle strength scoring using the Childhood Myositis Assessment Scale (CMAS), muscle enzyme testing, examination findings and associated co-morbidities). Global disease activity scores for patients/families and physicians were assessed by asking respondents to rate disease activity over the past week using a 10 point VAS scale.

Patients were in various stages of disease duration and severity at the time of enrollment. Those with incomplete data for the variables of physician and patient global activity assessment scores were excluded.

\section{Definition of discordance in global activity assessment scores}

Our primary outcome was patient/parent and physician global activity assessment scores, based on a standard 10-point VAS. There is no standardization regarding definitions for discordant or concordant scores when comparing global activity assessment scores between parents/patients and physicians [6]. Based on prior studies, we defined a greater than or equal to 2 point difference between a PF gVAS and MD gVAS as discordant $[10,11]$. PF gVAS and MD gVAS scores within 2 points were defined as concordant. Based on these definitions, discordant scores could have PF gVAS greater than MD gVAS (meaning the patient/parent rated the patient as having more disease activity compared to the physician) or could have MD gVAS greater than PF gVAS (meaning the physician rated the patient as having more disease activity compared to the patient/parent).

\section{Statistical analysis}

We assessed discordance between PF gVAS and MD gVAS, defined as at least a 2 point difference, and then evaluated factors associated with this discordance for each of two possible discordant scenarios: PF $>$ MD and $\mathrm{MD}>\mathrm{PF}$. For univariate associations, chi-square was used to compare categorical variables. Multivariate 
logistic regression analysis was applied to identify variable that were independent predictors of discordance with adjusted odds ratios and 95\% confidence intervals as displayed in a forest plot figure. Two-tailed values of $p<0.05$ were considered statistically significant. Statistical analysis was performed using the IBM SPSS software package (version 24.0, IBM Corporation, Armonk, NY).

\section{Results}

\section{Demographics and clinical characteristics}

From 2010 to 2014, 639 JDM patients were enrolled to the CLR, of which 563 patients had PF and MD gVAS data available for analysis. Mean age at enrollment was 10.6 years (range 6.9-14.7) with average age of onset 5.5 years (3.6-9.3). Most patients were female $(n=403$, $72 \%)$, Caucasian $(n=442,79 \%)$ and non-Hispanic $(n=$ $471,84 \%)$ (Table 1).

In this JDM cohort, 60 patients $(11 \%)$ had ongoing weakness described as moderate or severe, $37 \%(n=122)$ had a CMAS of $<48,52 \%(n=289)$ had a rash, $42 \%(n=$ 227) had ongoing nailfold capillaroscopy abnormalities, $10 \%(n=53)$ had calcinosis, and 18\% $(n=97)$ had abnormal muscle enzymes. Half of patients were receiving oral steroids $(n=261)$, while $14 \%$ were receiving IV pulse steroids $(n=69$; Table 1$)$.

\section{Comparison of global activity assessment score discordance}

Overall, 61\% $(n=344)$ of PF and MD gVAS scores were concordant (within 2 points of each other on 10-point VAS scale), $26 \%(n=149)$ were discordant with PF rating of gVAS $\geq 2$ points above (worse than) MD, and $12 \%$ $(n=70)$ were discordant with MD rating of gVAS worse than PF gVAS. Of the discordant scores $(39 \% ; n=219)$, $68 \%(\mathrm{n}=149)$ of PF rated their disease activity as worse than the MD, while $32 \%(n=70)$ of MD rated higher disease activity compared with PF (Table 1). The factors found to be significantly associated with discordance in each of the groups, as well as those with no discordance, are described in Table 2.

When PT VAS was $\geq 2$ points above MD (indicating poorer functioning/more severe disease), these patients had significantly worse CHAQ scores and more frequently reported poor quality of life $(p<0.01)$. When MD gVAS was $\geq 2$ points above PT gVAS, these patients had more frequent muscle enzyme abnormalities, worse proximal muscle weakness, rash, nail fold changes, calcinosis, higher percentage of joint involvement, and current IV pulse steroid treatment (all $p<0.01$ ). Other medications, including biologic medications and oral or subcutaneous methotrexate, were not significantly associated with discordance $(p=0.55,0.08$ and 0.07 , respectively). Demographic factors, including current age, age of onset, disease duration, gender, race/ethnicity, and income level, were also not associated with discordance in the VAS scores between patients/families and physicians (Table 2).

\section{Multivariable analysis of factors associated with discordance}

Multivariable logistic regression analysis of discordance in global activity assessment, where PF gVAS was rated as $\geq 2$ points higher than MD gVAS, included independent predictors of calcinosis (OR 2.1, 95\% CI: 1.2-4.2, $p=0.043$ ), CHAQ $>0.125$ (OR 2.0, 95\% CI: 1.2-3.0, $p=$ 0.004 ) and lower quality of life scores (good/very good vs excellent, OR 12.5, 95\% CI: 4.5-25, $p<0.001$; poor vs. excellent, OR 17.0, 95\% CI: 4.6-32, $p<0.001)$.

Multivariable logistic regression of discordance in global activity assessment, where MD gVAS was rated $\geq 2$ points higher than PF gVAS, found several significant independent predictors, including rash (OR 11.0, 95\% CI: 3.7-22, $p<0.001$ ), calcinosis (OR 3.3, 95\% CI: 1.4-8.2, $p=0.009$ ), nailfold capillaroscopy changes (OR 2.1, 95\% CI: $1.1-4.3, p=0.040)$, and patients receiving pulse steroids (OR 2.3, 95\% CI: 1.3-5.0, $p=0.039$ ).

This multivariable analysis is summarized in a forest plot, with the adjusted odds ratio of discordance and 95\% CI for each significant independent predictor (Fig. 1).

\section{Discussion}

There is a rapidly growing focus in healthcare regarding the importance of patient-centered care, with the goal of improving care that is most relevant to patients and families. This focus is especially important in chronic conditions, including juvenile dermatomyositis.

In this analysis of the JDM CLR, we found that in about $60 \%$ of cases, patient/parent and physician global assessments of disease activity were similar based on the concordance of their reported global activity scores. However, in approximately $40 \%$ of cases, there was significant discordance between the PF and MD reported gVAS scores. We also found that patients/families rated themselves as doing worse compared to their treating physicians two times more often than they rated themselves as doing better ( $26 \%$ vs. $12 \%)$, supporting our previous findings that patient/family perspectives vary from health care professionals in JDM [12].

The rate of discordance in this study highlights some limitations in this subjective measure of global disease activity. Discordance between patient/family and physician global activity assessment can lead to difficulty assessing the effectiveness of treatment, particularly in chronic conditions like JDM. In addition, significant discordance in global activity assessment is likely to be associated with lower patient/family satisfaction and decreased adherence to the recommended treatment regimen [13]. While it is possible that some of this 
Table 1 Demographic information for the patients with JDM in the CARRA Legacy Registry

\begin{tabular}{|c|c|c|c|c|}
\hline \multirow[b]{2}{*}{ Variable } & \multirow[b]{2}{*}{$\begin{array}{l}\text { Overall Cohort } \\
(N=563)\end{array}$} & \multicolumn{3}{|c|}{ Comparison of Global Assessment Scores } \\
\hline & & $\begin{array}{l}\text { Concordant gVAS: } \\
(N=344)\end{array}$ & $\begin{array}{l}\text { Discordant gVAS: PF gVAS > MD gVAS } \\
(N=149)\end{array}$ & $\begin{array}{l}\text { Discordant gVAS: MD gVAS > PF gVAS } \\
(N=70)\end{array}$ \\
\hline Current Age, years $\uparrow$ & $10.6(6.9-14.7)$ & $10.8(6.9-14.7)$ & $10.2(6.7-14.2)$ & $10.4(7.8-14.8)$ \\
\hline Age at Onset, years $\uparrow$ & $5.5(3.6-9.3)$ & $5.5(3.6-9.2)$ & $5.3(3.6-9.2)$ & $5.8(3.6-10.0)$ \\
\hline Disease Duration, years $\uparrow$ & $3.1(1.3-6.4)$ & $3.4(1.7-6.0)$ & $2.9(1.3-6.8)$ & $2.8(1.0-7.0)$ \\
\hline \multicolumn{5}{|l|}{ Gender, no. (\%) } \\
\hline Male & $160(28)$ & $99(29)$ & $43(29)$ & $18(26)$ \\
\hline Female & $403(72)$ & $245(71)$ & $106(71)$ & $52(74)$ \\
\hline \multicolumn{5}{|l|}{ Race, no. (\%) } \\
\hline Caucasian & $442(79)$ & $280(81)$ & $110(74)$ & $52(74)$ \\
\hline Black & $68(12)$ & $38(11)$ & $20(13)$ & $10(14)$ \\
\hline Asian & $20(4)$ & $11(3)$ & $7(5)$ & $2(3)$ \\
\hline Other & $33(6)$ & $15(5)$ & $12(8)$ & $6(9)$ \\
\hline \multicolumn{5}{|l|}{ Ethnicity, no. (\%) } \\
\hline Non-Hispanic & $471(84)$ & $287(83)$ & $126(85)$ & $58(83)$ \\
\hline Hispanic & $92(16)$ & $7(17)$ & $23(15)$ & $12(17)$ \\
\hline \multicolumn{5}{|l|}{ Income Level, no. (\%) } \\
\hline$<\$ 50,000$ & $162(36)$ & $90(33)$ & $53(44)$ & $19(34)$ \\
\hline$\geq \$ 50,000$ & $283(64)$ & $180(67)$ & $66(56)$ & $37(66)$ \\
\hline \multicolumn{5}{|l|}{ CMAS, no. (\%) } \\
\hline$<48$ & $122(37)$ & $74(35)$ & $29(38)$ & $19(44)$ \\
\hline$\geq 48$ & $211(63)$ & $139(65)$ & $48(62)$ & $24(56)$ \\
\hline \multicolumn{5}{|l|}{ Weakness, no. (\%) ${ }^{\text {a }}$} \\
\hline None or Mild & $494(89)$ & $311(92)$ & $130(90)$ & $53(76)$ \\
\hline Moderate or Severe & $60(11)$ & $28(8)$ & $15(10)$ & $17(24)$ \\
\hline \multicolumn{5}{|l|}{ Muscle Enzyme, no. (\%) ${ }^{a}$} \\
\hline Abnormal & $97(18)$ & $48(15)$ & $24(17)$ & $25(36)$ \\
\hline Normal & $446(82)$ & $284(85)$ & $117(83)$ & $45(64)$ \\
\hline \multicolumn{5}{|l|}{ JDM Rash, no. (\%) ${ }^{a}$} \\
\hline Yes & $289(52)$ & $160(47)$ & $68(47)$ & $61(87)$ \\
\hline No & $265(48)$ & $180(53)$ & $76(53)$ & $9(13)$ \\
\hline \multicolumn{5}{|l|}{ Nailfold Changes, no. (\%) ${ }^{a}$} \\
\hline Yes & $227(42)$ & $126(38)$ & $52(37)$ & $49(72)$ \\
\hline No & $313(58)$ & $206(62)$ & $88(63)$ & $19(28)$ \\
\hline \multicolumn{5}{|l|}{ Calcinosis, no. (\%) ${ }^{a}$} \\
\hline Yes & $53(10)$ & $18(5)$ & $21(15)$ & $14(20)$ \\
\hline No & $487(90)$ & $310(95)$ & $121(85)$ & $56(80)$ \\
\hline \multicolumn{5}{|l|}{ Gl-Cardiac, no. (\%) } \\
\hline Yes & $17(3)$ & $6(2)$ & $7(5)$ & $4(6)$ \\
\hline No & $546(97)$ & $338(98)$ & $142(95)$ & $66(94)$ \\
\hline \multicolumn{5}{|l|}{ Joint Involvement, no. (\%) ${ }^{a}$} \\
\hline Yes & $72(13)$ & $34(10)$ & $20(14)$ & $18(36)$ \\
\hline No & $481(87)$ & $306(90)$ & $124(86)$ & $51(64)$ \\
\hline
\end{tabular}

Pulse Steroids Use, no. (\%) ${ }^{a}$ 
Table 1 Demographic information for the patients with JDM in the CARRA Legacy Registry (Continued)

\begin{tabular}{|c|c|c|c|c|}
\hline \multirow[b]{2}{*}{ Variable } & \multirow[b]{2}{*}{$\begin{array}{l}\text { Overall Cohort } \\
(N=563)\end{array}$} & \multicolumn{3}{|c|}{ Comparison of Global Assessment Scores } \\
\hline & & $\begin{array}{l}\text { Concordant gVAS: } \\
(N=344)\end{array}$ & $\begin{array}{l}\text { Discordant gVAS: PF gVAS > MD gVAS } \\
(N=149)\end{array}$ & $\begin{array}{l}\text { Discordant gVAS: MD gVAS > PF gVAS } \\
(N=70)\end{array}$ \\
\hline Yes & $69(14)$ & $32(10)$ & $20(15)$ & $17(27)$ \\
\hline No & $441(86)$ & $283(90)$ & $113(85)$ & $45(73)$ \\
\hline \multicolumn{5}{|c|}{ Oral Steroids Use, no. (\%) ${ }^{\text {a }}$} \\
\hline Yes & $261(50)$ & $133(42)$ & $84(62)$ & $44(69)$ \\
\hline No & $258(50)$ & $187(58)$ & $51(38)$ & $20(31)$ \\
\hline $\mathrm{CHAQ} \uparrow$ & $0(0.0-0.63)$ & $0(0.0-0.38)$ & $0.38(0.0-1.09)$ & $0.13(0.0-0.88)$ \\
\hline CMAS Strength & $50(44-52)$ & $51(45-52)$ & $49(45-52)$ & $48(37-52)$ \\
\hline Pain Score & $1(0-3)$ & $0(0-2)$ & $2(0-5)$ & $0(0-2)$ \\
\hline \multicolumn{5}{|l|}{ QoL Score ${ }^{a}$} \\
\hline Excellent & $129(23)$ & $109(32)$ & $5(3)$ & $15(21)$ \\
\hline Good or Very Good & $395(71)$ & $217(64)$ & $129(88)$ & $49(70)$ \\
\hline Poor & $31(6)$ & $12(4)$ & $13(9)$ & $6(9)$ \\
\hline
\end{tabular}

a Significant univariate association. Discordance defined as at least a 2-point difference in gVAS

$\uparrow$ Data presented as median (interquartile range)

discordance could be related to differences in interpretation of the rating scale by patients/families and physicians, the wording of the question to the groups was identical and these measures have undergone validation testing [4]. It is therefore important to understand factors that contribute to discordance, as this may help us to identify better approaches to better capture patient/ family perspectives of disease burden and direct the development of better PROs for JDM in the future.

Our exploratory univariate analysis suggested some correlations of interest: poorer CHAQ and Quality of Life score are associated with worse PF gVAS, while elevated muscle enzymes, proximal muscle weakness, rash, arthritis and steroid use is associated with worse MD gVAS. However our multivariable regression analysis gives us some more useful insight into what may drive clinician and patient/family gVAS ratings, as we found certain independent predictors of discordance. Our findings suggest that clinicians may use clinical features, such as rash, nailfold capillaroscopy changes, calcinosis, and medications to inform their global assessment of disease, whereas patients/families may place a greater emphasis on PROs such as global assessment of disease on decreased function (based on CHAQ scores) and quality of life. It is possible that other patient-centered factors, such as fatigue and mental health, could also contribute to patient/family assessments of disease. Pain was not found to be a significant factor associated with discordance in our patient population, but overall pain scores were low in our cohort and pain may not have been a common enough symptom to identify discordance.

Interestingly, in multivariable analysis calcinosis was an independent factor that influenced discordance on gVAS in both directions. Though calcinosis is considered a measure of disease damage rather than activity according to IMACS, there is debate in clinical practice and the literature with regard to whether calcinosis represents disease damage and/or disease activity and it is a

Table 2 Factors associated with discordance ( $\geq 2$ point difference, with higher score indicating poorer functioning/more severe disease) in gVAS scores between PF and MD (all $p<0.01$ ), and those with no effect on discordance

\begin{tabular}{lll}
\hline Patient/Family gVAS score higher & Physician gVAS score higher & No effect on discordance \\
\hline Poorer CHAQ scores & Muscle enzyme abnormalities & Current age/age of onset \\
Poorer Quality of Life scores & Proximal muscle weakness & Disease duration \\
& Presence of rash/calcinosis & Gender \\
& Nailfold capillaroscopy changes & Race/ethnicity \\
& Joint involvement & Income level \\
Current use of steroids & CMAS score
\end{tabular}

Gastrointestinal/Cardiac involvement 


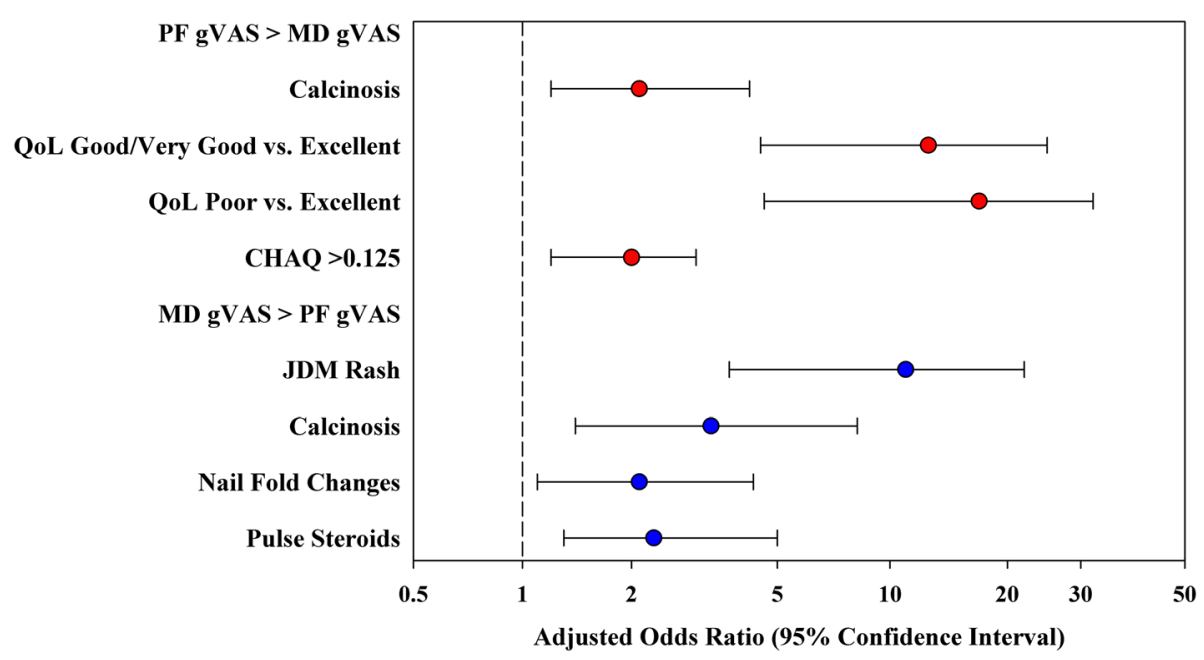

Fig. 1 Forest plot illustrating the adjusted odds ratios and 95\% confidence intervals for each significant independent predictor of PF and MD discordance in gVAS. Discordance is defined as at least a 2-point difference on the $0-10$ VAS scale. The red circles indicate predictors increasing the odds of discordance, where PF > MD. The blue circles denote predictors increasing the odds of discordance, where MD > PF

common practice to treat calcinosis with increasing immunosuppression [14-16]. This result highlights the important impact of calcinosis on the reported and perceived JDM disease activity by physicians as well as patients, and further evaluation into the factors driving this as an independent predictor of discordance in both directions would be an interesting topic for further study. This result also emphasizes the need to monitor and treat this important clinical manifestation of disease. Though we do not know the type and extent of calcinosis of patients from the CLR, we suspect that patients with calcinosis and worse PF gVAS scores likely had lesions that contributed to functional limitations, pain and concerns regarding physical appearance, in addition to other factors not measured in the registry.

The importance of capturing the patient/family perspective of disease activity in JDM cannot be minimized. In addition to the importance of including patient/family perspectives, PROs may be able to help better inform clinicians of disease activity in myositis, as it has in other conditions. For example in adult myositis, patients with reduced health related quality of life scores had lower muscle strength [17]. Dynamic repetitive muscle function has also been found to correlate with patientreported physical function [18].

Fortunately, international organizations conducting research in JDM, including IMACS and PRINTO, were prescient to include specific PROs as part of their disease core set measures, such as patient global assessment; however, there remains more work to be done in this field. As our work suggests, the extent to which these PROs capture the aspects of the disease that are important to patients/families has not been well studied, and these tools were originally developed with limited patient input. In addition, currently existing PROs were not developed specifically for inflammatory myositis. Outcome Measures in Rheumatology (OMERACT), an international initiative interested in outcome measures in rheumatology, has a myositis working group to develop, examine and validate PROs in adult myositis [19]. To improve the outcomes of our patients with JDM, it will be important to extend this work to patients with pediatric myositis in the future.

As with any study, there are limitations to our findings. Overall, patients enrolled in the CLR trended toward milder disease and the median disease duration in this cohort was short, which may limit the generalizability of the findings. In addition, since this was a cross sectional cohort, assessment of these measures and determination of concordance at specific time points (e.g., at diagnosis, remission, flares, etc.) was not possible. Differences in disease duration or severity could be expected to impact responses from patients and families; however, in our cohort there was no significant difference in disease duration between the physician and patient/family groups who had concordant gVAS or discordant gVAS in either direction. We also do not know if patients or parents filled out the PF gVAS. This could complicate interpretation of the results, since previous studies have shown differences in responses to patient reported outcome metrics when assessed by the patient or a family member/care giver [ 20 , 21]. It would have been interesting to assess differences in patient compared to physician scores, as well as patient compared to parent scores, and this would be an interesting topic for future study. Furthermore, it is possible that the gVAS score could be interpreted differently between different physicians; however, training on the use of these tools was available through CARRA and associated 
research groups, and previous studies have assessed interrater reliability of physician gVAS in inflammatory disorders with good results [22].

We were limited in the number of outcomes we could assess, and the CLR collected a limited number of PROs. Some PROs of particular importance, such as measures of fatigue, anxiety and depression, were not collected, which could potentially also impact gVAS score results. The current updated version of the CARRA Registry is more comprehensive and includes additional PROs, which should be incorporated into future studies.

\section{Conclusions}

In summary, we found a nearly $40 \%$ rate of discordance between patient/family and physician global activity assessment scores in patients with JDM enrolled to the CLR. Overall, worse patient/family scores were associated with worse PROs, while worse MD scores were associated with poorer objective measures of disease activity. Our findings suggest that PF and MD gVAS may often measure different facets of JDM disease activity and burden. Our work underscores the need to develop alternative relevant and valid patient-focused outcome measures that can be integrated into our overall assessment of patients with JDM, for use not only in clinical trials, but also in clinical decision-making and routine care of patients with JDM to improve future outcomes from this disease.

\section{Abbreviations}

CARRA: Childhood Arthritis and Rheumatology Research Alliance; CHAQ: Childhood Health Assessment Questionnaire; CMAS: Childhood Myositis Assessment Scale; gVAS: Global Disease Activity Score; IMACS: International Myositis Assessment and Clinical Studies Group; JDM: Juvenile Dermatomyositis; MD: Physician; OMERACT: Outcome Measures in Rheumatology; PF: Patient or Family; PRINTO: Pediatric International Trials Organisation; PROs: Patient Reported Outcome Measures; VAS: Visual Analog Scale

\section{Acknowledgements}

The authors wish to acknowledge CARRA and the ongoing Arthritis Foundation financial support of CARRA.

The CARRA Legacy Registry was supported by a grant from National Institute of Arthritis and Musculoskeletal and Skin Diseases of the National Institutes of Health under award Number RC2AR058934. The content is solely the responsibility of the authors and does not necessarily represent the official views of the National Institutes of Health. The CARRA Legacy Registry was also supported by CARRA, Friends of CARRA, the Arthritis Foundation, and the Duke Clinical Research Institute.

We would also like to thank all participants and hospital sites that recruited patients for the CARRA Registry. The authors thank the following CARRA Registry site principal investigators and research coordinators: L. Abramson, E. Anderson, M. Andrew, N. Battle, M. Becker, H. Benham, T. Beukelman, J. Birmingham, P. Blier, A. Brown, H. Brunner, A. Cabrera, D. Canter, D. Carlton, B. Caruso, L. Ceracchio, E. Chalom, J. Chang, P. Charpentier, K. Clark, J. Dean, F. Dedeoglu, B. Feldman, P. Ferguson, M. Fox, K. Francis, M. Gervasini, D. Goldsmith, G. Gorton, B. Gottlieb, T. Graham, T. Griffin, H. Grosbein, S. Guppy, H. Haftel, D. Helfrich, G. Higgins, A. Hillard, J.R. Hollister, J. Hsu, A. Hudgins, C. Hung, A. Huttenlocher, N. Ilowite, A. Imlay, L. Imundo, C.J. Inman, J. Jaqith, R. Jerath, L. Jung, P. Kahn, A. Kapedani, D. Kingsbury, K. Klein, M. Klein-Gitelman, A. Kunkel, S. Lapidus, S. Layburn, T. Lehman, C. Lindsley, M. MacgregorHannah, M. Malloy, C. Mawhorter, D. McCurdy, K. Mims, N. Moorthy, D. Morus,
E. Muscal, M. Natter, J. Olson, K. O'Neil, K. Onel, M. Orlando, J. Palmquist, M. Phillips, L. Ponder, S. Prahalad, M. Punaro, D. Puplava, S. Quinn, A. Quintero, C. Rabinovich, A. Reed, C. Reed, S. Ringold, M. Riordan, S. Roberson, A. Robinson, J. Rossette, D. Rothman, D. Russo, N. Ruth, K. Schikler, A. Sestak, B. Shaham, Y. Sherman, M. Simmons, N. Singer, S. Spalding, H. Stapp, R. Syed, E. Thomas, K. Torok, D. Trejo, J. Tress, W. Upton, R. Vehe, E. von Scheven, L. Walters, J. Weiss, P. Weiss, N. Welnick, A. White, J. Woo, J. Wootton, A. Yalcindag, C. Zapp, L. Zemel, and A. Zhu.

\section{Authors' contributions}

SK, HT and DZ made substantial contributions to conception and design, acquisition of data, analysis and interpretation of data, and drafting and critical revision of the manuscript. All authors read and approved the final manuscript.

\section{Funding}

The work of the authors of this study was supported, in part, by a small grant from the Childhood Arthritis and Rheumatology Research Alliance. The funding body had no impact on the study design, collection, analysis or interpretation of data, or in the writing of this manuscript.

\section{Availability of data and materials}

The datasets analyzed during the current study are available from the corresponding author on reasonable request.

\section{Ethics approval and consent to participate}

The Boston Children's Hospital IRB approved the work carried out in this study.

\section{Consent for publication}

Not applicable.

\section{Competing interests}

The authors declare that they have no competing interests.

\section{Author details}

'Division of Pediatric Rheumatology, Connecticut Children's Medical Center, 282 Washington Street, Hartford, CT 06106, USA. ${ }^{2}$ Department of Pediatrics, University of Connecticut School of Medicine, Farmington, CT, USA. ${ }^{3}$ Departments of Anesthesiology and Surgery, Boston Children's Hospital, Harvard Medical School, Boston, MA, USA. ${ }^{4}$ Pediatric Rheumatology, Benioff Children's Hospital and University of San Francisco Medical Center, San Francisco, CA, USA.

Received: 6 September 2019 Accepted: 6 January 2020

Published online: 15 January 2020

\section{References}

1. Rider $L G$, Nistala K. The juvenile idiopathic inflammatory myopathies: pathogenesis, clinical and autoantibody phenotypes, and outcomes. J Intern Med. 2016;280:24-38.

2. Black N, Jenkinson C. How can patients' views of their care enhance quality improvement? Br Med J. 2009;339:202-5.

3. Rider LG, Aggarwal R, MacHado PM, Hogrel JY, Reed AM, Christopher-Stine $\mathrm{L}$, et al. Update on outcome assessment in myositis. Nat Rev Rheumatol. 2018;14(5):303-18.

4. Rider LG, Werth VP, Huber AM, Alexanderson H, Rao AP, Ruperto N, Herbelin $L$, Barohn R, Isenberg D, Miller FW. Measures of adult and juvenile dermatomyositis, polymyositis, and inclusion body myositis. Arthritis Care Res. 2011;63(11):S118-57.

5. Rider LG, Aggarwal R, Pistorio A, Bayat N, Erman B, Feldman BM, et al. 2016 American College of Rheumatology/European league against rheumatism criteria for minimal, moderate, and major clinical response in juvenile dermatomyositis: an international myositis assessment and clinical studies group/Paediatric rheumatology inter. Ann Rheum Dis. 2017;76(5):782-91.

6. Desthieux C, Hermet A, Granger B, Fautrel B, Gossec L. Patient-physician discordance in global assessment in rheumatoid arthritis: a systematic literature review with meta-analysis. Arthritis Care Res. 2016;68(12):1767-73.

7. Eder L, Thavaneswaran A, Chandran V, Cook R, Gladman DD. Factors explaining the discrepancy between physician and patient global 
assessment of joint and skin disease activity in psoriatic arthritis patients. Arthritis Care Res. 2015;67(2):264-72.

8. Palmisani E, Solari N, Pistorio A, Ruperto N, Malattia C, Viola S, et al. Agreement between physicians and parents in rating functional ability of children with juvenile idiopathic arthritis. Pediatr Rheumatol. 2007;5:23.

9. Phillippi K, Hoeltzel M, Byun Robinson A, Kim S, Abramson LS, Anderson ES, et al. Race, income, and disease outcomes in juvenile Dermatomyositis. J Pediatr. 2017;184:38-44.

10. Smolen JS, Breedveld FC, Schiff MH, Kalden JR, Emery P, Eberl G, et al. A simplified disease activity index for rheumatoid arthritis for use in clinical practice. Rheumatol. 2003:42(2):244-57.

11. Khan NA, Spencer HJ, Abda E, Aggarwal A, Alten R, Ancuta C, et al. Determinants of discordance in patients' and physicians' rating of rheumatoid arthritis disease activity. Arthritis Care Res. 2012;64(2):206-14.

12. Tory HO, Carrasco R, Griffin T, Huber AM, Kahn P, Robinson AB, et al. Comparing the importance of quality measurement themes in juvenile idiopathic inflammatory myositis between patients and families and healthcare professionals. Pediatr Rheumatol. 2018;16:28.

13. Jackson CA, Clatworthy J, Robinson A, Horne R. Factors associated with non-adherence to oral medication for inflammatory bowel disease: a systematic review. Am J Gastroenterol. 2010;105:525-39.

14. Orandi AB, Baszis KW, Dharnidharka VR, Huber AM. Hoeltzel and for the CARRA juvenile myositis subgroup. Assessment, classification and treatment of calcinosis as a complication of juvenile dermatomyositis: a survey of pediaric rheumatologists by the childhood arthritis and rheumatology research alliance (CARRA). Pediatr. Rheumatol. 2017:15:71.

15. Bellutti Enders F, Bader-Meunier B, Baildam E, Constantin T, Dolezalova P, Feldman BM, et al. Consensus-based recommendations for the management of juvnile dermatomyositis. Ann Rheum Dis. 2017;76(2):32940

16. Hinze $\mathrm{CH}$, Speth F, Oommen PT, Haas JP. Current management of juvenile dermatomyositis in Germany and Austria: an online survey of pediatric rheumatologists and pediatric neurologists. Pediatr Rheumatol. 2018;16:38.

17. Poulsen KB, Alexanderson H, Dalgård C, Jacobsen S, Weile L, Diederichsen LP. Quality of life correlates with muscle strength in patients with dermatoor polymyositis. Clin Rheumatol. 2017;36(10):2289-95.

18. Alexanderson H, Regardt M, Ottosson C, Munters LA, Dastmalchi M, Dani L, et al. Muscle strength and muscle endurance during the first year of treatment of polymyositis and dermatomyositis: a prospective study. $J$ Rheumatol. 2018;45(4):538-46.

19. Park JK, Mecoli CA, Alexanderson H, Regardt M, Christopher-Stine L, CasalDomínguez M, et al. Advancing the development of patient-reported outcomes for adult myositis at OMERACT 2016: an international Delphi study. J Rheumatol. 2017;44(11):1683-7.

20. Shaw KL, Southwood TR, McDonagh JE. Growing up and moving on in rheumatology: parents as proxies of adolescents with juvenile idiopathic arthritis. Arthritis Care Res. 2006;55(2):189-98.

21. Sattoe JNT, Van Staa A, Moll HA. The proxy problem anatomized: childparent disagreement in health related quality of life reports of chronically ill adolescents. Heal Qual Life Outcomes. 2012;10:10.

22. Pincus T, Castrejon I, Chua J, et al. AB1173 Physician visual analog scale estimates for overall global assessment, inflammation, damage, and distress to assess patients and support clinical decisions in routine rheumatology care: analysis of inter-rater reliability. Annals of the Rheumatic Dis. 2017;76: 1466.

\section{Publisher's Note}

Springer Nature remains neutral with regard to jurisdictional claims in published maps and institutional affiliations.

Ready to submit your research? Choose BMC and benefit from:

- fast, convenient online submission

- thorough peer review by experienced researchers in your field

- rapid publication on acceptance

- support for research data, including large and complex data types

- gold Open Access which fosters wider collaboration and increased citations

- maximum visibility for your research: over $100 \mathrm{M}$ website views per year

At BMC, research is always in progress.

Learn more biomedcentral.com/submissions 